\title{
Dynamic Cell-Based MAC Protocol for Target Detection Applications in Energy-Constrained Wireless Networks
}

\author{
Sonia Waharte and Raouf Boutaba \\ University of Waterloo, School of Computer Science \\ 200, University Av. West, Waterloo, Canada N2L 3G1 \\ \{swaharte, rboutaba\}@bbcr. uwaterloo.ca
}

\begin{abstract}
Today's advances in sensor technology make it feasible to embed sensing, communication and computation capabilities in small untethered nodes. However, node lifetime is still severely restricted by the limitations of power supply. By improving power consumption efficiency of sensor node operations, a sensor node's lifetime can be significantly extended. A well designed MAC protocol can achieve this goal by minimizing the amount of data transmitted through the network. In scenarios where data retrieval operations are infrequent and localized (e.g. target detection applications), pre-configuring an entire sensor network is detrimental to power conservation. Therefore, restricting data gathering operations to nodes with valuable information can significantly reduce energy consumption. In this paper, we propose and evaluate a new MAC protocol, and demonstrate the advantages of our scheme for specific types of applications.
\end{abstract}

\section{Introduction}

Recent technological advances have enabled the development of tiny devices embedding communication, sensing and computation capabilities. These devices are self-organized after deployment and coordinate themselves to perform some common tasks, such as sensing the environment, retrieving accurate data, and gathering data for further processing. Sensor networks are envisioned to find applications in diverse fields such as environment monitoring, battlefield surveillance, target tracking, traffic analysis, etc. They are intended to be deployed in any environment, outdoor or indoor, and cover large-scale areas, often making it infeasible to replace the nodes' limited power supply. As some applications (environment monitoring, fire detection, etc.) require sensor nodes to have a lifetime in the range of several months or years, one solution to extend the lifetime of the sensor nodes is to reduce the number of messages sent through the network during data gathering operations and during the network self-organization process. In the recent literature, the most commonly proposed solution relies on the concept of cluster formation [1], [2], [3], [4]. Some nodes in the sensor network are elected to act as cluster heads and collect data from the other nodes located 
in their close vicinity. This configuration is particularly adapted to applications that require constant data retrieval from all the nodes in the network. However, cluster formation is not advantageous for applications needing only infrequent sensing operations on localized events.

Consider an application such as target tracking. Only the sensor nodes in proximity to the tracked target should participate in the data gathering process. Thus, the overall network lifetime can be significantly improved if we consider the formation of cells (groups of nodes located in the same vicinity), created in reaction to the detection of a specific stimulus, instead of a proactive network organization. Such approach is also more adapted to the tracking of moving targets.

We propose a Dynamic Cell-based MAC Protocol (DCP) whose principle is the following: using a multi-frequency mechanism, a self-elected cell head coordinates the data transmission of a set of neighboring nodes in order to minimize data collision. This paper describes the features of DCP and its performance in comparison to a traditional Time Division Multiple Access scheme. In this paper, we focus only on local node organization and we are therefore not concerned with the routing aspect of data transmission.

The paper is organized as follows. After a discussion of related works in Section 2, we present the design and implementation of DCP in Section 3. In Section 4, we show the results of the conducted simulations and demonstrate the advantages of DCP for the targeted application scenarios.

\section{Related Works}

An important research effort in wireless sensor networks has been conducted to reduce energy consumption of sensor nodes operations in order to increase the network's lifetime. Several mechanisms have been proposed to achieve this goal by turning on/off the sensor nodes or by implementing data gathering processes aiming at decreasing the overall traffic load. In this paper, we focus on the latter mechanism.

Several data gathering mechanisms have been designed for wireless sensor networks. They essentially adopt the same clusters formation approach excepted PEGASIS [5], which proposes to construct a chain among the nodes in the sensor network, with the election of a random leader node responsible for the transmission of the gathered data to the destination station.

Among the clustering approaches, a cyclic scheme has been proposed by LEACH (Low-Energy Adaptive Clustering Hierarchy) [6]. At the beginning of each round, each node decides to elect itself as a cluster head with a probability directly related to its energy level. To avoid collision, a transmission schedule is then established between the cluster head and the nodes in its cluster. Instead of a proactive configuration of the network, our proposal tries to avoid the synchronization problem through a reactive cell formation.

Chevallay, Van Dyck and Hall [1] took a different approach by limiting the number of nodes per cluster (they suggested 8 ). The cluster heads then form the 
backbone of the network. The clusters can be merged according to a predefined criterion called attractiveness (geographical proximity of the cluster heads). This protocol assumes the existence of node and group identifiers. DCP eliminates the problem of node identifiers using the multi-frequency approach described in the next section.

Krishnan and Starobinski proposed in [3] a node organization based on a growth budget defined by an initiator node (the growth budget corresponds to the number of children a node is allowed to have). Two algorithms for clusters formation are proposed: in the first one, Algorithm Rapid, the initiator node sends a message to its neighbors, which, according to the allocated budget, forward this message to their neighbors except the parent node. The process stops when the budget is exhausted. If a node is a leaf node, the allocated budget is wasted. In the second algorithm, Algorithm Persistent, a system of reallocation of unutilized budgets was introduced as an improvement of the first algorithm. In our approach, the node acting as initiator node does not have to be aware of the number of existing neighbor nodes.

Zhang and Arora proposed an algorithm for self-organization and self-healing of wireless sensor networks based on a cellular structure [7]. The destination station is defined as the center of the first cell. The algorithm begins by determining the heads of the neighboring cells. The cell heads thus selected run the same algorithm and the process goes on until the discovery of the whole network. Nodes are supposed to be able to detect the locations of other nodes, facilitating the process of self-healing in case of failure of the cell heads. After selection of the cell heads, the remaining nodes decide to join the cell with which the communication is the least energy-consuming (the cell head is the nearest geographically). This scheme and DCP explore the same idea of cell formation but in different ways. Whereas [7] assumes a fixed cellular topology, we propose a dynamic cell creation with the self-election of the cell head at the location where the targeted event takes place.

Some schemes based on the dynamic formation of cells have been recently proposed. However, they often rely on the assumption of the presence of nodes with enhanced capabilities, which are able to assume the role of cluster head [8]. In this paper, we intend to alleviate such constraints by considering the election of what we call cell coordinators at close vicinity to the event being monitored without any assumption on their capabilities. In addition, only the nodes with relevant information transmit data to the cell coordinator.

\section{Dynamic Cell-Based MAC Protocol (DCP)}

DCP is best suited for applications with sporadic data retrievals, such as safety applications (threat detection), surveillance systems or alarm generation. We believe that due to the infrequency of data retrieval operations, triggered by specific localized phenomena, only a limited number of nodes should be involved in the data gathering process. Such localized event does not justify the cost of pre-configuring an entire sensor network. For these applications, dynamic network organization is more suitable than traditional clustering approaches. 
The idea underlying DCP is as follows: after detecting a specific event, a node informs its neighbors of its intention to report the results of its sensing operations to the remote destination station. Through this action, it automatically elects itself as the cell coordinator and becomes responsible for organizing data transfers from its neighbors, via a registration process. We devise a transmission schedule among registered nodes based on a time slot scheme. One major advantage of our model is that only the neighbor nodes willing to transmit information to the cell coordinator have to go through the registration process. As the cell coordinator is elected only for one data gathering process, the process of cell formation is repeated every time a node has information to report to the remote destination station.

We assume that the nodes in proximity of each other have correlated and often identical data to send to the destination station. Indeed, the perception of the same event occurring in a localized area will not differ significantly from one sensor node to another. Thus, if a sensor node dies (energy depletion, failure, etc), its loss will not affect the accuracy of the data sent by the surrounding nodes to the destination station.

DCP presents two apparent advantages. First, it is non-cyclic and therefore requires no synchronization. Second, we do not have any loss of bandwidth due to unused time slot because the node organization is dynamic, reactive, and involves only nodes with relevant information to send.

\subsection{DCP Design}

At any time, upon detection of an external event requiring an immediate report to the destination station, a node can initiate the cell creation process. We adopte a multi-frequency approach with simultaneous registrations to reduce the overhead entailed by node identification. Nodes are identified by the frequency they choose to register on. Moreover, for the implementation of our protocol, we assume that a control frequency $f$ is set before the deployment of the sensor network. The control frequency is used to avoid multiple cell formations at the same time, in the same location. Prior to electing itself as a cell coordinator, a node has to listen to the control channel for a predetermined period of time. If the channel is idle, it then deduces that it can proceed with the cell formation process. A group of frequencies $f_{1}, \ldots, f_{N}$ is also set for the registration process. The data gathering process can be divided into three phases (Figure 1):

1. The self-elected cell coordinator informs its neighbors that it has data to report to the destination station. This information is conveyed to its neighbors via a TR_INFO packet sent on the control frequency (Figure 1 (a)).

2. The nodes located in the cell (range of emission of the cell coordinator) register themselves by replying with a TR_RESP message, if they have information to send. Each node chooses a random frequency among the group specified in the TR_INFO message (Figure $1(\mathrm{~b})$ ). To address the problem of multiple nodes colliding on the same frequency, each node begins its transmission after a random backoff time. During this period, the nodes listen 


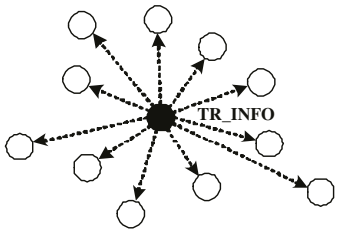

(a) Invitation process

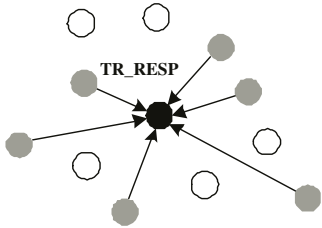

(b) Registration Phase

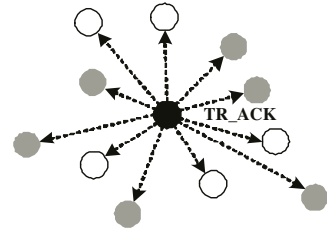

(c) Registration Confirmation

Fig. 1. Data gathering mechanism

to the chosen channel. If the channel is busy, a node immediately chooses another channel and resumes its waiting period. If the new chosen channel is also busy, the node then considers that the information it wants to transmit is redundant (as a number of other nodes in close vicinity will transmit the same information), and switches to sleep mode.

During the transmission of TR_RESP signals, the cell coordinator scans the range of frequencies to determine which frequency is in use (if a frequency is used, that means that a node wants to register).

3. The cell coordinator builds a list of the frequencies used and sends it back to the nodes in a TR_ACK packet (Figure 1 (c)).

Finally, the registered nodes wait until their allocated time slot to wake up and transmit their data.

\subsection{Dimensioning of the Number of Frequencies}

In wireless environments, where the number of frequencies available is highly restricted, loss of bandwidth due to data collision is a critical problem that can be partially alleviated by the implementation of collision avoidance mechanisms such as the CSMA/CA MAC protocol. In multi-frequency approaches, further constraints are introduced in that the same frequency should not be allocated to neighboring cells (inter-cell collision) or to neighboring nodes in the same cell (intra-cell collision). Moreover, as the number of frequencies per group and the number of frequency groups directly impact the throughput available for data transmission, a tradeoff has to be made between these two factors.

Inter-cell Collisions. If several nodes elect themselves as cell coordinators in the same geographical area, formation of overlapping cells may occur. Hence, if the same frequency is selected by several neighboring cells, the chance of data collision will increase dramatically. In order to avoid this situation, different frequency groups have to be defined, so that each cell will choose a group different from its neighbors, such as no inter-cell collision occurs. To achieve this objective, we need to determine the exact number of frequency groups needed, which is mainly dependent on the cell distribution in the sensor field. By considering the worst case, illustrated in Figure 2, we can derive an upper bound for the 

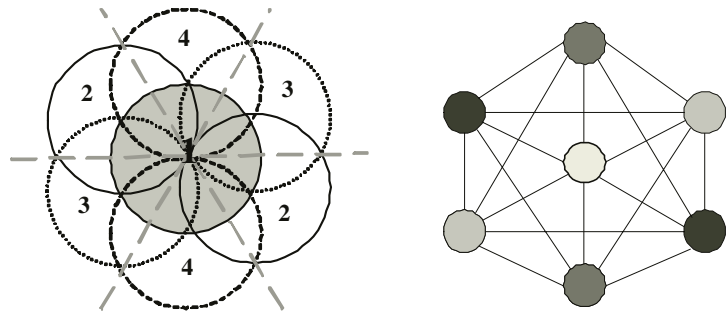

Fig. 2. Overlapping cells - This is similar to the Graph-Coloring problem, where two adjacent cells (graphically represented with a disk) must not transmit on the same frequency band

maximum possible number of adjacent cells and then choose an average number of frequency groups. Actually, by using 4 different frequency bands, it is possible to avoid inter-cell interference.

Some inter-cell collisions may appear during the cell formation (collisions on TR_INFO packets). The use of random backoff time before the transmission of a TR_INFO packet reduces the probability of collision but cannot totally prevent it from happening. One feasible solution is to implement a process of negative acknowledgments. While listening to the control channel, if a node (any node in the cell but the cell coordinator which can not detect a collision while transmitting) detects a collision, it sends on the control channel a busy tone warning the cell coordinators that a collision occurred on the TR_INFO packets. As some nodes may not be aware of the collision, the cell coordinators have to send another busy tone on the control channel to inform every node in the cell that a collision occurred and that a new cell formation process has to be started again.

Intra-cell Collisions. The probability of intra-cell collision is defined as the probability of two or more nodes deciding to choose the same frequency for the registration process (we suppose that the choice of a frequency is random).

In the following, we assume that the network density is such that the number of frequencies available will always be greater than the number of nodes willing to register in a specific cell. Let us consider a group of $\mathrm{k}$ sensor nodes located geographically in the same cell, $F=\left\{f_{i}, i=1 . . N\right\}$ the set of frequencies available for the registration process and $C=\left\{c_{i}, i=1 . . k\right\}$ the frequencies selected by the nodes.

The probability of collision can be defined as:

$$
P(\text { collision })=1-P\left(D_{k, N}\right)
$$

where $D_{k, N}$ is the event where k nodes choose $\mathrm{k}$ different frequencies among $\mathrm{N}$, such that:

$$
D_{k, N}=\bigcap_{i=1}^{k} F_{i, N}
$$


where $F_{i, N}$ is the event that node $i$ chooses a frequency different from node $j$, for all $j<i$, among a group of $\mathrm{N}$ frequencies available.

We can then deduce the probability of collision:

$$
\begin{aligned}
& P(\text { collision })=1-P\left(F_{k, N} \mid D_{k-1, N}\right) P\left(D_{k-1, N}\right) \\
& P(\text { collision })=1-P\left(D_{1, N}\right) \prod_{i=2}^{k} P\left(F_{i, N} \mid D_{i-1, N}\right)
\end{aligned}
$$

This probability, dependent on the number of frequencies available $N$ and on the number of nodes willing to register $k$, can be expressed as:

$$
P(\text { collision })=1-\frac{N !}{(N-k) ! N^{k}}
$$
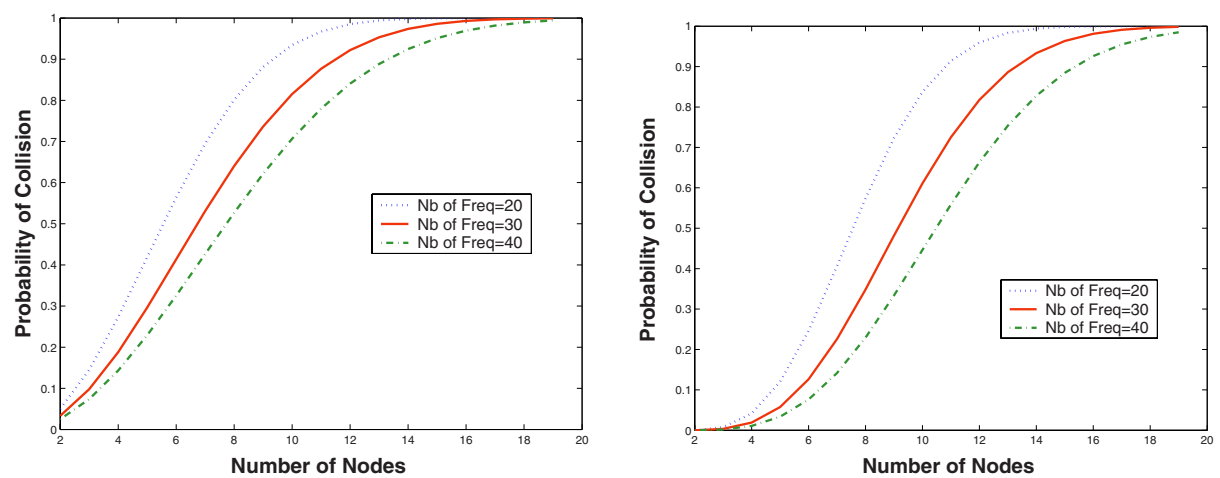

Fig. 3. (a) Probability of collision (b) Probability of collision with the "second chance" mechanism

In Figure 3 (a), by varying the number of frequencies per frequency group, we illustrate the probability of intra-cell collisions based on the number of nodes. We observe that as the number of nodes in a neighborhood increases, regardless of the number of frequencies available for use, the probability of collision tends to unity. To have a probability of collision below $50 \%$ with a number of frequencies equal to 30 requires that the number of nodes in a neighborhood be below 6 . To relax this constraint, we introduce a "second chance" mechanism. In this scheme, every node (that has information to report) attempts to register itself twice. During the first attempt, if the randomly chosen frequency appears to be busy, the node will randomly choose another frequency and repeat the registration process.

Using the same method as for the previous calculation of the probability of collision, we define the probability of collision when using the "second chance" mechanism as:

$$
P(\text { collision })=P\left(C_{2} \mid C_{1}\right) P\left(C_{1}\right)
$$


where $C_{2}$ is the event where at least one over $\mathrm{k}$ nodes experiences a collision during the second round of the registration process and $C_{1}$ is the event where at least one over $\mathrm{k}$ nodes experiences a collision during the first round of the registration process. Then, an approximation can be derived as:

$$
\begin{gathered}
P(\text { collision })=P\left(C_{2}\right) P\left(C_{1}\right) \\
P(\text { collision })=\left(1-P\left(D_{1, N-1}\right) \prod_{i=2}^{k}\left(P\left(F_{i, N-1} \mid D_{i-1, N-1}\right)\right)\right) \\
\left(1-P\left(D_{1, N}\right) \prod_{i=2}^{k} P\left(F_{i, N} \mid D_{i-1, N}\right)\right)
\end{gathered}
$$

This probability of collision, shown in Figure 3 (b), can be expressed as:

$$
P(\text { collision })=\left(1-\frac{(N-1) !}{(N-k) !(N-1)^{k-1}}\right)\left(1-\frac{N !}{(N-k) ! N^{k}}\right)
$$

Tradeoff. Let Scan_Time denote the time to scan the range of frequencies, Detect_Time denote the time to detect energy on a channel, Hop_Time denote the time to switch from one frequency to another and $\mathrm{N}$ the number of frequencies so that:

$$
\text { Scan_Time }=\text { Detect_Time } * N+\text { Hop_Time } *(N-1)
$$

The number of frequencies per group has a direct impact on the scan time, and hence, on the overall registration delay. An under-proportioned number of frequencies would lead to a high level of collisions and a limited number of registered nodes. At the opposite end, an over-proportioned number of frequencies would allow more registration possibilities but a longer registration process.

If we consider 30 frequencies per group and 21 frequency groups ( 5 times the minimum required plus 1 group for the control channel), the bandwidth available $^{1}$ per frequency group becomes:

$$
\frac{\text { ISM band }}{\text { Number of Frequency Groups }}=\frac{26}{21}=1.238 \mathrm{MHz}
$$

As there is no synchronization between the cells, the probability that two cells would decide to transmit at the same time is negligible. In order for two cells to transmit simultaneously, they must first contend for the control channel and then choose the same frequency group. This scenario happens with a probability of the order of $10^{-6}$ for an initial contention window of 31 .

The local synchronization during data transmission is maintained thanks to the geographical proximity of all the nodes in a cell. Actually, every node in a cell is at most $20 \mathrm{~m}$ away from the cell coordinator. Upon reception of the TR_ACK

\footnotetext{
${ }^{1}$ We consider the $902-928 \mathrm{MHz}$ Industrial, Scientific and Medical (ISM) band.
} 
packet, the nodes go to sleep and wake up during their allocated time slots. For each time slot, the maximum overlapping period lasts 66ns (propagation time for a distance of $20 \mathrm{~m}$ at $300000 \mathrm{~km} / \mathrm{s}$ ). Knowing that the precision of a GPS system is around 200ns [9], we can consider that the cell coordinator acts as a beacon and synchronizes all the nodes in the cell by sending first the TR_INFO packet and next the TR_ACK packet.

\section{Performance Evaluation}

\subsection{Energy Consumption Evaluation}

We present our analysis and evaluate our framework according to the radio propagation model described in [2]. The energy $E_{T x}$ to transmit a packet and $E_{R x}$ to receive a packet can be stated as:

$$
\begin{gathered}
E_{T x}=l E_{\text {elec }}+l \epsilon d^{2} \\
E_{R x}=l E_{\text {elec }}
\end{gathered}
$$

where $E_{\text {elec }}=50 \mathrm{~nJ} / \mathrm{bit}, l$ is the packet size, $\epsilon=100 \mathrm{pJ} / \mathrm{bit} / \mathrm{m}^{2}$ and $d$ is the transmission distance.

By applying these formulas to our protocol, we obtain:

$$
\begin{gathered}
E_{\text {coordinator }}=E_{T x, T R_{-} I N F O}+E_{T x, T R_{-} A C K}+N E_{R x, S c a n} \\
E_{\text {non-coordinator }}=E_{R x, T R_{-} I N F O}+E_{R x, T R_{\_} A C K}+E_{T x, T R \_R E S P} \\
E_{\text {non-coordinator }}=E_{R x, T R_{-} I N F O}+E_{R x, T R_{\_} A C K}+E_{T x, T R \_R E S P}
\end{gathered}
$$

Let us suppose that we have $\mathrm{k}$ nodes in the cell and $\mathrm{N}$ frequencies available for the registration process, the total energy consumption can thus be expressed as:

$$
\begin{gathered}
E_{\text {total }}=E_{T x, T R_{-} I N F O}+E_{T x, T R_{-} A C K}+N E_{R x, S c a n}+(k-1)\left(E_{R x, T R \_I N F O}\right. \\
\left.+E_{R x, T R_{\_} A C K}+E_{T x, T R \_R E S P}\right) \\
E_{\text {total }}=\left(k l_{T R_{-} I N F O}+k l_{T R_{-} A C K}+(k-1) l_{T R_{-} R E S P}+N l_{S c a n}\right) E_{\text {elec }} \\
+\left(l_{T R \_I N F O}+l_{T R_{\_} A C K}+(k-1) l_{T R \_R E S P}\right) \epsilon d^{2}
\end{gathered}
$$

In this analysis, we use a 100x100 meters network, and vary the number of nodes per cell. For simplicity, we consider that the transmission distance can not exceed 20 meters. The area covered by one cell is thus in the order of $1200 \mathrm{~m}^{2}$. Thus a minimum of 8 simultaneous cells (called clusters) can be formed. We compare three types of network organizations: a single cell, three cells and 8 cells, with varying network density (we suppose a uniform node distribution).

Intuitively, the result obtained is not surprising. The energy saving obtained from a single cell formation (DCP 1 cell) is advantageous when the data retrieval 


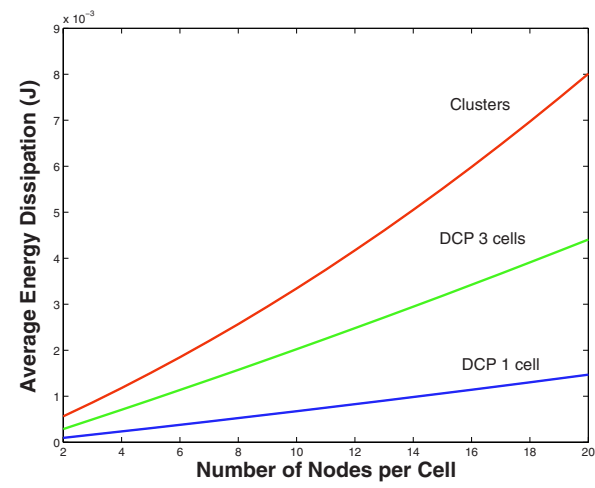

Fig. 4. Energy dissipation evaluation

is infrequent. When considering three cell formations, we can see that the energy consumption increases. But the gain of dynamic cells formations compared to a global network organization is largely dependent on the size of the network (this analysis only considers a very small number of clusters). This model is best adapted for scenarios where the data retrieval is localized and infrequent. As previously mentioned, some real world applications like target tracking exhibit these characteristics. A cell of $1200 \mathrm{~m}^{2}$ is large enough for such applications. Moreover, the advantage of our model is more apparent in large scale networks.

\subsection{Simulation Parameters}

We evaluate the performance of our model by simulations using OMNET ++ [10], an object-oriented discrete event simulator. We compare our design to a cell formation based on a TDMA scheme without acknowledgment process. The initial schedule is established by contention according to the IEEE 802.11 specifications. For simplicity, we kept the same designation for the name of the packets, even if the packets exchanged in both models are different. In the TDMA approach, TR_INFO packets are composed of a header and a 2-byte cell coordinator identifier. TR_RESP packets also include a header, the cell coordinator identifier and the sender node identifier. TR_ACK packets are composed of a header, the cell coordinator identifier and the list of IDs of the registered nodes.

We set our simulation parameters as follows:

- The time to detect energy on a frequency is set to Detect_Time $=15 \mu \mathrm{s}$ [11].

- The hop time to change from one frequency to another is set to Hop_Time= $224 \mu \mathrm{s}$ [11].

- We consider only the case of one cell formation.

- DCP is based on a combination of FDMA, TDMA and Direct Sequence Spread Spectrum (to reduce narrowband interference and noise effects) with a nominal data rate fixed at $112 \mathrm{kbps}$. 
- For the cell formation process based on TDMA, we consider a Direct Sequence Spread Spectrum over 21 frequency bands of $1.2 \mathrm{MHz}$ each $^{2}$. The nominal data rate is fixed at $112 \mathrm{kbps}$.

- Time intervals are set to $15 \mu \mathrm{s}$.

- Packet headers are set to 25 bytes.

In DCP, at the beginning of each registration process, the nodes randomly choose a frequency and transmit a busy tone on this frequency after a random backoff time. In the simulations, we set the contention window to 44 . Thus, we have 45 time slots ( 1 time slot $=15 \mu \mathrm{s}$ ) for the backoff period, which corresponds to 3 times the average number of neighbor nodes in case of a node density of 0.01 nodes $/ \mathrm{m}^{2}$.

We evaluate our protocol according to two criteria. First, we study the registration delay in order to demonstrate the effectiveness of our scheme for timesensitive applications such as target detection. Second, we estimate the number of registered nodes, a critical indicator for the accuracy of the sensing operations. In fact, it is necessary to ensure that the number of nodes successfully registered is above a certain threshold (the determination of this threshold is application-dependent).

\subsection{Simulation Results}

By increasing the number of nodes in a cell, we show that DCP still performs better than the TDMA scheme in terms of delay, while maintaining a high level of node registration rate. We define the Registration Delay as the global setup time of the registration process (from sending the TR_INFO packet to the reception of the last TR_ACK packet) and Number of Nodes as the total number of nodes in the cell, including the cell coordinator. Hence the maximum number of registered nodes at most equals to the total number of nodes minus 1 .

Figure 5(a) depicts the average registration delay as defined previously and the corresponding number of nodes in the cell. The top curve represents the registration delay for the TDMA-based cell formation (referred as Cluster). The simulation is performed over multiple iterations, where the number of nodes in the cell is incremented per iteration. For each iteration, the simulation is repeated 100 times. The registration delay increases almost linearly with the number of nodes. This increase in registration delay corresponds to the time needed to transmit the TR_RESP packet to the cell coordinator. The bottom curve depicts the registration delay for DCP. We only observe a slight increase in the delay corresponding to the increase in the size of the TR_ACK packets (the TR_ACK packets include the ID/frequencies of the registered nodes). DCP performs better than a classic TDMA-based cell formation when the number of nodes in the cell increases above 3 nodes. This result can be explained by

\footnotetext{
${ }^{2}$ The number of orthogonal codes being limited, the occurrence of inter-cells interference can be reduced by the addition of a frequency band division technique, whose description is beyond the scope of this paper.
} 

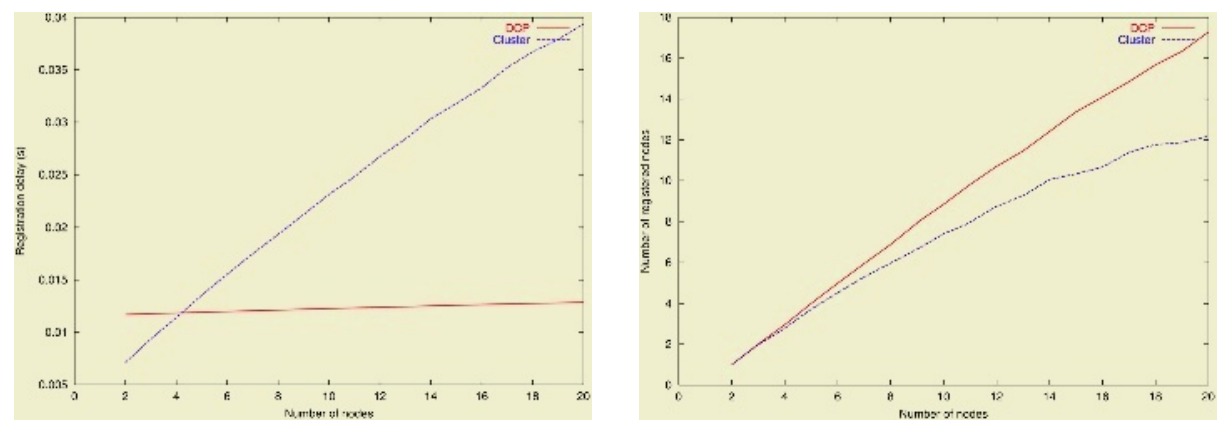

Fig. 5. (a) Average Delay (b) Average number of registered nodes

the implementation of the scanning process in DCP, which involves a minimum registration delay that is rapidly amortized when the number of neighbors is above 2 .

Figure 5(b) depicts the average number of registered nodes, as well as the total number of nodes in the cell. The top curve represents the average number of registered nodes for the DCP model. Similar to the above case, we run the simulations 100 times and compute the average. The bottom curve represents the average number of registered nodes for a cluster formation without acknowledgment process. The "second chance" mechanism implemented in our protocol allows the nodes two attempts to register and accounts for the performance gain over the cluster formation.

In order to analyze the efficiency of our protocol, we implemented a cluster of 15 nodes, and ran the simulation for 200 iterations. We only consider the registration process without data transmission. The results of the simulations are summarized in Table 1 and in Table 2.

The analysis of the standard deviation for the registration delay and for the number of registered nodes gives insights on the stability of both protocols. DCP appears to be much more stable than the TDMA scheme, and performs significantly better in terms of number of registered nodes and registration delay. This is due to the fact that the fluctuations of the TDMA scheme are directly corre-

Table 1. Analysis of the TDMA scheme behaviour over a large number of simulation runs for 15 nodes per cell

\begin{tabular}{|c||c|c|}
\cline { 2 - 3 } \multicolumn{1}{c|}{} & $\begin{array}{c}\text { Number of Registered } \\
\text { Nodes }\end{array}$ & $\begin{array}{c}\text { Registration Delay } \\
(\mathbf{m s})\end{array}$ \\
\cline { 2 - 3 } & 10.285 & 31.751185 \\
\hline Mean & 14 & 35.986 \\
\hline Maximum & 5 & 26.086 \\
\hline Minimum & 2.21547209 & 2.466758021 \\
\hline Standard Deviation & 0.307042937 & 0.341868729 \\
\hline $\mathbf{9 5 \%}$ Confidence Interval & 0.403523385 & 0.449292298 \\
\hline $\mathbf{9 9 \%}$ Confidence Interval & &
\end{tabular}


Table 2. Analysis of DCP behaviour over a large number of simulation runs for 15 nodes per cell

\begin{tabular}{|c||c|c|}
\cline { 2 - 3 } \multicolumn{1}{c|}{} & $\begin{array}{c}\text { Number of Registered } \\
\text { Nodes }\end{array}$ & $\begin{array}{c}\text { Registration Delay } \\
(\mathbf{m s})\end{array}$ \\
\cline { 2 - 3 } Mean & 13.395 & 12.579045 \\
\hline Maximum & 14 & 12.622 \\
\hline Minimum & 10 & 12.338 \\
\hline Standard Deviation & 0.756197177 & 0.05369 \\
\hline $\mathbf{9 5 \%}$ Confidence Interval & 0.104801592 & 0.007440913 \\
\hline $\mathbf{9 9 \%}$ Confidence Interval & 0.137732832 & 0.009779031 \\
\hline
\end{tabular}

lated to the number of collisions that occurred during the registration process. For DCP, the slight fluctuations are due to the number of unregistered nodes (decreasing the size of the TR_ACK packet). Concerning the number of registered nodes, our model guarantees a better performance compared to a classic cluster formation. The minimum number of registered nodes for a cell of 15 nodes (including the cell coordinator) is 10 for DCP, whereas for the cluster formation this number drops to 5 . The $95 \%$ and $99 \%$ confidence intervals illustrate the improvement of DCP over the TDMA scheme.

Overall, DCP performs better than the TDMA scheme both in terms of registered nodes and in terms of delay, particularly when the number of nodes in the network increases. More precisely, our protocol initially outperforms the cluster formation approach based on a TDMA scheme. This performance gain wanes over time, because the performance of cluster formation is amortized over several rounds. The advantage of our model is the dynamic and rapid cell formation.

\section{Conclusion}

Given that some applications need only infrequent sensing operations, we proposed a MAC protocol based on the creation of cells in the vicinity of the targeted event. The proposed Dynamic Cell-based MAC Protocol adopts a reactive approach, with a data gathering process that is triggered by the detection of a specific stimulus requiring an immediate report to the destination station. The advantages of our protocol are to reduce bandwidth loss due to unused time slots in TDMA schemes and to provide a faster cell formation while avoiding data collisions. At the same time, our multi-frequency approach does not require node identification. Moreover, no global synchronization is necessary because the cell coordinator is used as a beacon node to organize the data transmission process.

The preliminary analysis of energy consumption gives an estimate of the amount of energy dissipation during the cell formation process and supports the theory that if the data retrieval is localized and infrequent, a complete network organization is expensive. The results of the conducted simulations show that DCP performs better than traditional TDMA approaches in terms of delays 
and collisions reduction. The sending of tones on a frequency range during the registration process reduces the overall delay and decreases packet header size by avoiding the exchange of node identification.

\section{References}

1. Chevallay, C., Dyck, R.V., Hall, T.: Self-organization protocols for wireless sensor networks. In: Conference on Information Sciences and Systems. (2002)

2. Heinzelman, W., Chandrakasan, A., Balakrishnan, H.: An application-specific protocol architecture for wireless microsensor networks. In: IEEE Transactions on Wireless Communications. Volume 1. (2002) 660-670

3. Krishnan, R., Starobinski, D.: Message-efficient self-organization of wireless sensor networks. In: IEEE Wireless Communications and Networking Conference. (2003)

4. Mirkovic, J., Venkataramani, G., Lu, S., Zhang, L.: A self-organizing approach to data forwarding in large-scale sensor networks. In: IEEE International Conference on Communications. ICC 2001. Volume 5. (2001) $1357-1361$

5. Lindsey, S., Sivalingam, K.: Data gathering algorithms in sensor networks using energy metrics. IEEE Transactions on Parallel and Distributed Systems 13 (2002) 924-935

6. Catterall, E., Laerhoven, K.V., Strohbach, M.: Self-organization in ad hoc sensor networks: an empirical study. (2002) http://www.comp.lancs.ac.uk/ strohbach/.

7. Zhang, H., Arora, A.: GS3: scalable self-configuration and self-healing in wireless networks. In: Proceedings of the twenty-first annual symposium on Principles of distributed computing. (2002)

8. Chen, W.P., Hou, J., Sha, L.: Dynamic clustering for acoustic target tracking in wireless sensor networks. In: 11th IEEE International Conference on Network Protocols. (2003) $284-294$

9. Elson, J., Estrin, D.: Time synchronization for wireless sensor networks. In: Proceedings of the 15th International Parallel and Distributed Processing Symposium., IEEE (2001) 1965-1970

10. OMNET++ simulator: ("http://whale.hit.bme.hu/omnetpp/")

11. IEEE Std 802.11 Information Technology- telecommunications And Information exchange Between Systems-Local And Metropolitan Area Networks-specific Requirements-part 11: Wireless Lan Medium Access Control (MAC) And Physical Layer (PHY) Specifications: (1997) 